\title{
Influence of HeartMath Training Programme on Physiological and Psychological Variables
}

\author{
Lucy H. Field, L. ${ }^{1,2}$, Stephen D. Edwards ${ }^{3}$, David J. Edwards ${ }^{3}$ \& Sarah E. Dean ${ }^{1}$ \\ ${ }^{1}$ School of Life Science and Education, Staffordshire University, Stoke-on-Trent, United Kingdom \\ ${ }^{2}$ Liverpool John Moores University, Liverpool, United Kingdom \\ ${ }^{3}$ Psychology Department, University of Zululand, KwaDlangezwa, South Africa \\ Correspondence: David J. Edwards, University of Zululand, Private Bag X1001, KwaDlangezwa, 3886, South \\ Africa. E-mail: edwards.davidjohn@gmail.com.
}

Received: October 2, 2017 Accepted: November 17, 2017 Online Published: January 8, 2018

doi:10.5539/gjhs.v10n2p126 URL: https://doi.org/10.5539/gjhs.v10n2p126

\begin{abstract}
This study investigated biofeedback using heart rate variability (HRV) and psychometric measures. An original, mixed, quantitative and qualitative, within subjects, pre-test and post-test design was employed to evaluate the influence of a HeartMath training programme on measures of HRV, sense of coherence, mindfulness and relaxation in a convenience sample of 13 participants with age range 26-62 $(\mathrm{M}=41.46, \mathrm{SD}=14.39)$ years. Participants were required to attend a three-hour workshop and complete 30 minutes of HeartMath training that included 5 different biofeedback sessions. Paired sample t-tests indicated significant differences on all outcome variables with medium to large effect sizes. Qualitative findings revealed positive and informative experiences of taking part in the research. Integrated findings supported and extended previous studies attesting to the value of HeartMath training.
\end{abstract}

Keywords: HeartMath, training programme, biofeedback, heart rate variability, coherence

\section{Introduction}

Public health promotion programmes recognise stress as one of the prominent worldwide causes of premature death and it has been identified as the health epidemic of the 21st century (Dillon, Kelly, Robertson, \& Robertson, 2016). It is predicted that up to $60-80 \%$ of visits to the doctor are stress related including physical health complaints such as high blood pressure, cardiovascular disease, back pain, digestive problems, poor sleep and metabolic syndrome. In addition to pharmacology, stress reduction is reportedly achievable with various health promoting methods, including specific lifestyle and mind-body techniques, such as optimum physical activity of 150 minutes a week, breathing, meditation and psychophysiological biofeedback, through improved conscious control of physiological processes, for example through instruments measuring HRV as in the present study (Childre, Martin, Rozman, \& McCraty, 2016).

One recent approach to stress management is positive psychology, originally pioneered by Seligman and Csikszentmihalyi (2000). A positive psychology approach underlies the HeartMath system of self-regulation techniques used in stress management (Edwards, 2015; McCraty \& Shaffer, 2015). This views stress as a half empty bottle of negative energy that needs filling with positive energy, including excited, happy, peaceful and contented feelings, which initiate the release of beneficial hormones in the body such as the growth hormone dehydroepiandrosterone or DHEA. In terms of the positive psychology approach taken in the present study, stress was viewed as a pervasive phenomenon that appears physiologically in the form of lowered HRV, which is associated with decreased autonomic nervous system balance, decreased parasympathetic activity, decreased physiological coherence levels accompanied by increased sympathetic nervous system activity and increased levels of cortisol that can be measured in the body for up to eighteen hours after any perceived threat, as contrasted to the positive DHEA. From a psychological perspective, the effects of stress are apparent in negative emotions and their accompanying feelings of anxiety, depression, anger and confusion, and decreased sense of coherence, relaxation and mindfulness (Childre et al., 2016; Institute of HeartMath, 2014).

HeartMath techniques use the heart as point of entry to facilitate neural identification of changes in the pattern of afferent cardiac signals sent to the brain. Related research has revealed associated dynamic, systemic activity at 
various levels; neurochemical, bio-electromagnetic, hormonal, biophysical and cognitive (McCraty, Atkinson, Tomasino, \& Bradley, 2009). Skill acquisition of HeartMath techniques is facilitated through the use of HRV and heart rhythm coherence feedback training, heart focussed breathing and intentional generation of associated positive emotional feelings, emotional imagery and remembered wellness (McCraty \& Zayas, 2014). A practical, energetic approach underlies the techniques (Edwards, 2015). Emphasis is on awareness of energy depletion, renewal and resilience in preparing for challenges, as well as shifting and resetting feelings after challenges, through sustained, regular HeartMath practice.

Coherence is a key orientation concept, in its various connotations, logical argumentation, systemically related parts, biophysical and electromagnetic phase relationships, including psychophysiological coherence, which occurs during positive emotional states when heart, breath, blood pressure and other physiological rhythms become synchronized. It is characterized by a heart rhythm pattern of elevated amplitude in low frequency heart rate variability of around $0.1 \mathrm{~Hz}$, indicating harmony between sympathetic and parasympathetic divisions of the autonomic nervous system. It is experienced as a state of relaxed alertness, which sportspersons describe as "being in the zone" (McCraty et al., 2009). Although previous research has established that positive emotions are independently associated with psychophysiological coherence, heart focussed breathing at about 5-7 breath cycles per minute and/or 10 second cardio-respiratory rhythm remains a practical, first step in most tools. This conscious slowing of breath facilitates respiratory sinus arrhythmia (RSA), a dynamic, naturally occurring, physiological mechanism, whereby heart rate increases during inhalation and decreases during exhalation, which in turn facilitates identification and cultivation of a particular positive emotion (McCraty \& Zayas., 2014).

In pursuit of its central vision of facilitating personal, social and global coherence, the HeartMath Institute has pioneered integral, heart focussed research in neuroscience, cardiology, physiology, biochemistry, bioelectricity, physics and psychology (Institute of HeartMath, 2014). Its vast research library contains numerous rigorous studies conducted by members of the institute as well as independent researchers and organizations that attest to the effectiveness and value of the system. Various empirical studies have indicated that psychophysiological coherence is associated with improved positive emotional states, sense of coherence and mindfulness. Although physiological research has distinguished between relaxation and positive emotions such as appreciation, this has not yet been tested psychometrically. The research aimed to answer the question: How does targeted biofeedback training affect HRV, and perceptions of mood, coherence, relaxation and mindfulness? Quantitatively, it was hypothesized that the dependent variables; relaxation, sense of coherence, mood, mindfulness and HRV would improve and that this improvement would be qualitatively endorsed by participants' experiences.

\section{Method}

\subsection{Design}

This study had both a quantitative and a qualitative component. The quantitative aspect had a within subject design. Recent guidelines have recommended that when designing Heart Rate Variability Biofeedback (HRV-BF) interventions, within participant designs have the benefit and opportunity of measuring individuals pre-and post-intervention, which reduce 'the high inter-individual variations and the complex interactions influencing HRV' (Quintana \& Heathers, 2014). The independent variable was time, which had two levels: pre-and post-intervention. This study considered five dependent variables: stress, coherence, mindfulness, relaxation and HRV. Following the intervention, participants were invited to take part in a short interview to determine their experiences of using the HeartMath technology, which was analysed using thematic analysis.

\subsection{Participants}

A prospective power analysis was conducted to determine the required sample size. According to Clark-Carter (2010), for a large effect size (Cohen's $d$ between 0.7 and 0.8 ) and a power of $0.8,13$ participants were required. Thirteen participants were recruited for the study, 11 females and 2 males, age range for the participants was 26-62 $(\mathrm{M}=41.46, \mathrm{SD}=14.39)$ years. Participants volunteered by responding to an advert on Facebook. All participants lived in the North West of England, were middle class, employed and White British, 7 of whom were married and 6 of whom were single. The inclusion criteria consisted of individuals over the age of 18 with no declared major physical or mental health concerns. The exclusion criteria consisted of anyone with an existing heart condition, which may affect the baseline HRV measurements.

\subsection{Ethics}

Ethical approval was granted from the relevant Universities Ethics Committees. All participants provided written consent regarding the use of their information and were provided with an information sheet as to the research. Participants were guaranteed confidentiality and advised as to their right to withdraw from the research at any 
stage. Participants were informed they could have their data and could see their data throughout the research process.

\subsection{Measures}

Four questionnaires were used in the quantitative component of the research to measure mood, coherence, mindfulness and relaxation. Mood was assessed using a shortened version of the Profile of Mood States questionnaire (POMS) called the Brief Assessment of Mood States (BAMS) to measure changes in affective states (McNair, Lorr, \& Doppleman, 1971). The scale has six items and is used to assess five different mood states including anxiety, frustration, depression, fatigue and vigour. The scale has been shown to be valid and reliable alternative to the longer POMS (Bourgeois, LeUnes, \& Meyers, 2010). Participants were asked to rate their mood on a scale of 0-4, from not at all to extremely, for example, how angry do you feel? How fatigued do you feel? Total score is calculated by adding the scores and amending one score with a reverse item for feelings of vigour. A higher score indicates a higher level of negative emotions and stress.

The Sense of Coherence measure consisted of a shortened nine item version of Antonovsky's (1987) scale, with a Cronbach alpha reliability coefficient of .79. Antonovsky's (1987) original scale has three subscales, which measure the degree to which persons perceive their world as manageable, meaningful and predictable. The shortened version used in the present study has been shown to demonstrate high internal reliability and concurrent validity when assessed against Antonovsky's original 29 item measure (Klepp, Mastekaasa, Sorensen, Sandanger, \& Kleiner, 2007). Participants' reported their feelings in relation to items such as, "Do you have the feeling that you don't really care about what goes on around you?" on a nine item, seven point Likert scale anchored by the terms, "very often" and "very seldom". Total score is calculated by adding the scores and reversing the score for two negatively worded items. A higher score indicates a higher level of coherence.

Relaxation was assessed by the Relaxation Inventory (RI) which has been found to be valid and reliable (Crist, Rickard, Prentice-Dunn, \& Barker, 1989). This scale lists items which are used to assess the effects of relaxation training. Participants were asked to rate their feelings on a Likert scale from 1 to 9 and rate the items, for example, 'I feel very calm'. Total score is calculated by adding the scores from all items. A higher score indicates a higher level of relaxation.

The Freiburg Mindfulness Inventory (FMI) (Walach, Buchheld, Buttenmüller, Kleinknecht, \& Schmidt, 2006) includes thirty items and has been found to be sensitive to changes in mindfulness following intervention and been shown to be psychometrically sound with an internal consistency of Cronbach alpha $=.93$. Participants were asked to circle Rarely, Occasionally, Often, Almost always on all items. For example, 'I am open to the experience of the present moment', 'I sense my body, whether eating, cooking, cleaning or talking' and 'When I notice an absence of mind, I gently return to the experience of the here and now'. Total score is calculated by adding the scores from all items, a higher score indicates a higher level of mindfulness.

\subsection{Apparatus}

HRV measures of biofeedback were used as an indicator of physiological stress and coherence (Edwards, 2014). HRV measures of cardiac vagal tone can be calculated by using power spectrum analysis that measures the variation of the beat-to-beat intervals in the heart (Edwards, 2014). The emWavePro Plus consists of PC based software, a USB sensory unit that is attached to the computer and a wired ear clip. The ear clip clips over the participant's earlobe and uses a small amount of infrared light to measure blood flow in the ear. The software is used to measure and record HRV to provide values of low, medium and high physiological coherence and display the feedback on the screen to participants. The emWavePro Plus software sessions can be saved to a participant database or exported to third party software platform. A Dictaphone was used to record interviews with participants post-intervention. See table 1 below for interview schedule.

Table 1. Interview schedule

Questions
How did you find doing the five games in the training programme?
What was good about the training programme and what impact did it have on you?
Were you able to reach the 'state of coherence'? And if so how did it feel?
What have you learnt about ways of managing stress?
Is there anything you will do differently in the future as a result of taking part in the study?




\subsection{Procedure}

All participants were screened for appropriateness in relation to the inclusion and exclusion criteria. Suitable candidates were sent an information sheet and consent form prior to the study and the researcher answered any questions. Each participant was separately invited to attend a three-hour workshop at Staffordshire University, in the United Kingdom, which included a structured programme with regular breaks. Participants were required to complete four psychological outcome measures and a 10 minute HRV coherence assessment pre- and post intervention. This took place in a controlled quiet environment conducive to relaxation. Participants were shown the sensor that was placed on their ear and familiarised with the sensation that would cause. The sensor was removed and they were asked to fill in the four questionnaires. Participants were then asked to sit upright with feet firmly on the floor and hands in the lap. They were told to maintain this position for 5 minutes before baseline recording as recommended by Quintana and Heathers (2014) to encourage standardised measurement and acclimatization to the recording environment (Laborde, Mosley, \& Thayer, 2017). Participants were invited to sit upright and relax for 10 minutes while a sensor was attached to their ear to obtain a baseline assessment of HRV before any training took place. Using the HeartMath technology, the three-hour intervention taught individuals techniques for improving physiological coherence as measured by HRV. Participants were shown 'the quick coherence technique' and three stages of 'effective coherence training'. The first step was raising the participants' awareness of the physiology of their body via the involuntary HRV and connection to mind. In the second step, participants were trained in the ability to alter their physiology by learning to control their HRV through slower breathing and positive emotions. Finally, participants were educated to reach an optimal HRV state (Ratanasiripong, Park, Ratanasiripong, \& Kathalae, 2015). Participants were asked to practice the coherence technique for 10 minutes or until they had gained at least $60 \%$ level of coherence. They then moved on to HeartMath software, which provided biofeedback training exercises that appear as 'games'. Participants were required to complete 4 additional training games; garden game, rainbow game, my inspiration and the balloon game. The training games required individuals to use their HRV training, with higher levels of coherence and optimum HRV related to increased success in the games. Levels of HRV coherence were displayed in the right bottom corner of the screen. This revealed the percentage of coherence and the traffic light indicator; red = low coherence; orange $=$ medium coherence and green $=$ high coherence. The researcher guided each participant throughout the training and supported instruction to enable participants to achieve coherence using a defined protocol. After all training games were completed, resting HRV was then measured for 10 minutes post-intervention.

\subsection{Data Analysis}

Using Statistical Package for the Social Sciences (SPSS), a series of t-tests were carried out comparing changes in five dependent variables, pre-and post HRV-BF training. Assumption checks on all data were met, including, sensible and missing values, outliers, homogeneity of variance and normal distribution. A paired-samples t-test was carried out comparing pre- and post-intervention scores on each dependent variable. Significance levels were set at the conventional 5\% level. In addition, the effect sizes were calculated to measure the magnitude of all outcome variables. These were calculated by hand using Cohen's $d$. Thematic analysis was chosen to analyse the qualitative data. No pre-existing coding frame was utilised to try and minimise any influence of the researchers' preconceptions. However, it is acknowledged that the authors' theoretical and epistemological commitments are likely to impact on some level of interpretation (Braun \& Clarke, 2006). A five-stage protocol was followed completing the analysis as recommended by Braun and Clarke (2006). These steps include: Phase 1; getting to know the data, Phase 2; generating initial codes, Phase 3; searching for themes, Phase 4; reviewing themes and Phase 5; defining and naming themes. Each step was revised and data was re-read many times which is recommended for quality analysis (Braun \& Clarke, 2006). Central concepts were highlighted and formulated using a thematic map that led to the generation of themes.

\section{Results}

\subsection{Quantitative Findings}

Quantitative findings are summarized in Table 2 and their respective interpretations follow. Table 2 Pre-test and Post-test Mean, Standard Deviations (SD), Probability and Effect size for each measure POMS (Mood), SOC (sense of coherence), FM (mindfulness), RI (relaxation) and HRV coherence. HRV coherence levels were calculated using percentage change in HRV coherence level pre- and post training intervention. The data were obtained from the emWave ProPlus HeartMath assessment technology which has been shown to be a valid and reliable measurement tool to identify changes in HRV pre-post intervention (Institute of HeartMath, 2014). Single and double asterisks respectively indicate significance at $5 \%$ and $1 \%$ levels. 
Table 2. Means, Standard Deviations, Probability and Effect sizes of psychological and psychological measures

\begin{tabular}{lllllll}
\hline Measure & Pre-test & SD & Post-test & SD & Probability & Effect size \\
\hline POMS & 7.08 & $(4.80)$ & 3.08 & $(2.06)$ & $.002^{* *}$ & 0.83 \\
SOC & 34.23 & $(6.18)$ & 43.92 & $(8.76)$ & $.001^{* *}$ & 1.57 \\
RI & 187.15 & $(47.08)$ & 237.38 & $(20.34)$ & $.000^{* *}$ & 1.07 \\
FM & 36.92 & $(11.34)$ & 40.46 & $(9.47)$ & $.040^{* *}$ & 0.31 \\
HRV coherence & 40.18 & $(4.65)$ & 49.33 & $(13.03)$ & $.02^{*}$ & 1.97 \\
\hline
\end{tabular}

The findings of this current study reveal significant changes from pre-test to post-test in each dependent variable following HRV-BF intervention. The mean estimate of the POMS (mood variable) prior to intervention was 7.08 $(\mathrm{SD}=4.80)$ and post-intervention, $3.08(\mathrm{SD}=2.06)$, was statistically significant, $\mathrm{t}(12)=3.80, \mathrm{p}<0.05$, indicating that participants reported feeling less stressed following the intervention. Cohens (1988) effect size of 0.83 was large. The mean estimate of the RI (relaxation variable) prior to intervention was $187.15(\mathrm{SD}=47.08)$ and post-intervention was $237.38(\mathrm{SD}=20.34)$. This was statistically significant, $\mathrm{t}(12)=4.85, \mathrm{p}<0.01$, with a very large effect size of 1.07 indicating that participants reported improved levels of relaxation following the intervention. The mean estimate of the FM (mindfulness variable) prior to intervention was $36.92(\mathrm{SD}=11.34)$ and post-intervention was $40.46(\mathrm{SD}=9.47)$. This difference was statistically significant, $\mathrm{t}(12)=2.30, \mathrm{p}<0.05$, indicating that participants reported improved levels of relaxation following the intervention. The effect size was 0.31 , which according to Cohen (1988) is small to medium. The mean estimate of the SOC (sense of coherence variable) prior to intervention was $34.23(\mathrm{SD}=6.18)$ and post-intervention was $43.92(\mathrm{SD}=8.76)$. This difference was statistically significant, $\mathrm{t}(12)=4.40, \mathrm{p}<0.05$, with a very large effect size of 1.57 indicating that participants reported improved sense of coherence following the intervention. This finding was supported by a significant difference in physiological levels of HRV pre-post intervention, $t(12)=2.47, p<0.05$. The results all showed greater coherence and higher HRV levels post-intervention, which are indicators of improved functioning of the autonomic nervous system. The effect size was 1.97, which according to Cohen (1988) was very large.

\subsection{Qualitative Findings}

Qualitative analysis of results revealed 3 main themes: Appreciating the positive visual aspects of HeartMath, learning to retrain one's breathing, and improving self-regulation and psychophysiological coherence. Detailed findings follow.

\subsubsection{Theme 1: Appreciating the Positive visual Aspects of HeartMath}

Participants reported positive experiences of using the HeartMath technology, including the simplicity of the system and the use of visual images. Sarah reported 'I really enjoyed doing the visualisation ones like the garden and the rainbow one'. Participants reported to appreciate the scenes of nature which induced feelings of calmness and relaxation. Richard stated 'I found that I think because a lot of the exercises had nature involved in them water, stream, rainbows... like nice positive things that it made me feel really calm'.

\subsubsection{Theme 2: Learning to Retrain One's Breathing}

This theme was consistent throughout the data as the majority of participants reported to be unaware of the impact of breath and levels of stress prior to HRV-BF training. The data suggested that participants were learning breathing techniques for the first time and the training intervention appeared to improve this skill. In particular, most participants reported that the HeartMath technology enabled them to be more aware of their breath and that noticing breathing style was important. Ross stated 'Breathing is a key sign and noticing your breathing, and how to breathe and then notice how to bring your breathing back in a calm soothing manner'.

\subsubsection{Theme 3: Improving Self-Regulation and Psychophysiological Coherence through HeartMath}

Improving self-regulation and psychophysiological coherence through HeartMath appeared to be the main theme integrated throughout all the transcripts. Participants reported that a benefit of engaging in the training programme was the awareness of being in coherence. Sally used the term '...regulated... like everything was... yeah nice pleasant' and Beverly stated 'It got me in tune with what's going on with me erm. right here and now really'. Participants appeared to be aware both psychologically and physiologically when they entered a state of coherence 'when I was in coherence I felt serene'. Collectively, the themes provide evidence to suggest that individuals found the training, education interventions effective in raising awareness of aspects of both physiological and psychological components of stress. The incentivisation to continue with the breathing techniques is also noted 
throughout the transcripts. It is also clear that the nature of the games and biofeedback they received encouraged participants to continue to practice their self-regulation techniques.

\section{Discussion}

Integrated findings from both the qualitative and quantitative components of the research provided strong evidence to support the effectiveness of using HeartMath technology to improve HRV, coherence, mindfulness and relaxation. Results supported the research hypothesis with regard to significant differences in all five dependent variables following HRV-BF training. Large effect sizes were found in the current study for physiological HRV, sense of coherence, relaxation and mood, which support the recent meta-analysis by Goessl, Curtiss and Hofmann (2017). The findings of the present research confirm previous quantitative studies that have found HRV-BF interventions beneficial for improving coherence, heart rate variability and reducing stress in healthy populations (Edwards, 2014; McCraty et al., 2015).

The present study extended findings of other studies indicating associations between HeartMath practice and improved positive emotional states, perception of mindfulness and relaxation (Edwards, 2014; McCraty et al., 2015; Reiner, Atkinson, \& McCraty, 2008). This contemporary study provides evidence that by using measures of $\mathrm{HRV}$ it is possible to quantify the dynamic measure of stress and how this variable is reactive to current emotional states and changes in breath (Lemaire, Wallace, Lewin, de Grood, \& Schaefer, 2011). Collectively, the results from this current study, together with the literature, provide evidence that biofeedback interventions offer a promising contemporary approach to promoting self-regulation as a form of stress management, with improved measures of both physiological and psychological health (McCraty et al., 2015; Edwards, 2014).

The results from the present study contradicted other HRV-BF research studies that have not found significant differences in HRV following intervention (Whited, Larkin, \& Whited, 2014). Inconsistent findings may reflect differences in methodology regarding the intensity and frequency of self-regulation techniques taught in the intervention. Difference in findings may also reflect various non-specified variables, e.g. trainer trainee relationship and amount of trainee self-directedness. For example, the current study and findings from the literature that have used a thorough training programme with face-to face contact with the trainer had generally revealed significant psychological and physiological differences (McCraty et al., 2015; Edwards, 2014). It is hypothesised that self-management with a short training time might not be as effective as more guided training programmes due to lower levels of self-efficacy (van der Zwan, de Vente, Huizink, Bögels, \& de Bruin, 2015).

The present findings support previous qualitative literature in both meditation and biofeedback studies that have found increased awareness, insight and adaptive behavioural change following intervention (Benson, 1996, 2000; Kabat-Zinn, 2003). Collectively, the present study and current literature highlight that biofeedback interventions using HeartMath technology reveal transferability of themes including positive experiences of taking part, improved awareness, self-regulation and a better understanding of the mind-body connection (Edwards, 2014; McCraty et al., 2001). However, due to limited qualitative studies and small sample sizes, any generalisability should be treated with caution.

A limitation of this study was the unequal gender distribution. There were only two men in the study compared to 11 women and therefore results may not be generalizable to men. Gender differences have been found in previous research and it also well known that men do not seek support as freely as women (Tamres, Janicki, \& Helgeson, 2002). Limitations of this study also include lack of control group and subsequently, the significant differences reported in the psychological variables may also reflect social desirability and the experimenter effect. Recommendations for future research would include randomised control trials, the gold standard for robust interventions, to compare the effectiveness of different self-management techniques on reducing stress and improving psychological and physiological variables that compare both within and between analyses (Edwards, 2014). A strength of the current research was that no participants dropped out at any point in the research and participants completed all parts of the experiment and questionnaires leading to a full data set. This may be due to its non-invasive, non-threating and person centered nature (van der Zwan et al., 2015).

With the structure of national health services changing, over-burdened, under resourced and pushed to capacity, there is a drive for individuals to improve their self-management. Subsequently primary care providers could actively promote self-regulation skills and include system wide initiative to be proactive and promote individual wellness (Lemaire et al., 2011). Although biofeedback might not be suitable for all individuals, this method of self-management is suggested to be cost effective in the long term by reducing the number of individuals who continually present in GP surgeries and hospitals as a result of chronic stress, however future analysis will need to examine cost implications. Further longitudinal research is needed to examine the efficacy of HRV training to see if there are any sustaining benefits on psychological variables and physiological measures of HRV. There is now 
substantial evidence that biofeedback interventions can effectively reduce psychological and physiological measures of stress and that such interventions are acceptable and effective across a variety of both mental and physical health conditions (McCraty et al., 2014).

\section{Conclusion}

The findings of the present study provide consistent evidence that the HeartMath training programme was both feasible and effective in improving heart rate variability, physiological coherence, sense of coherence, relaxation and mindfulness. Further studies with larger samples, mixed method designs and more detailed analysis of measures from the HeartMath technology should be undertaken. This study provides an exciting opportunity for positive health psychology to implement such interventions to make an impact on the global epidemic of stress and facilitate local and global health promotion.

\section{Acknowledgements}

This work is based on research supported by the University of Zululand, South Africa and the South African National Research Foundation (NRF). Any opinion, finding and conclusion or recommendation expressed in this material is that of the author(s) and the NRF does not accept any liability in regard thereto.

\section{Competing Interests Statement}

The authors declare that there are no competing or potential conflicts of interest.

\section{References}

Antonovsky, A. (1987). Unravelling the mystery of health. San Francisco, CA: Jossey Bass.

Benson, H. (1996). Timeless healing. London, England: Scribner.

Benson, H. (2000). The relaxation response. New York, NY: HarperCollins.

Bourgeois, A., LeUnes, A., \& Meyers, M. (2010). Full-scale and short-form of the Profile of Mood States: A factor analytic comparison. Journal of Sport Behavior, 33(4), 355-376.

Braun, V., \& Clarke, V. (2006). Using thematic analysis in psychology. Qualitative Research in Psychology, 3(2), 77-101. https://doi.org/10.1191/1478088706qp063oa

Childre, D. L., Martin, H., Rozman, D., \& McCraty, R. (2016). Heart intelligence. Connecting with the intuitive guidance of the heart. HeartMath, CA: Waterfront Press.

Clark-Carter, D. (2010). Quantitative psychological research: The complete student's companion (3rd ed.). Leicester, England: The British Psychological Society.

Crist, D. A., Rickard, H. C., Prentice-Dunn, S., \& Barker, H. R. (1989). The relaxation inventory: Self-report scales of relaxation training effects. Journal of Personality Assessment, 53(4), 716-726. https://doi.org/10.1207/s15327752jpa5304_8

Dillon, A., Kelly, M., Robertson, I. H., \& Robertson, D. A. (2016). Smartphone applications utilizing biofeedback can aid stress reduction. Frontiers in Psychology, 7. https://doi.org/10.3389/fpsyg.2016.00832

Edwards, S. D. (2014). Effects of biofeedback training on physiological coherence, health and spirituality perceptions: Sport and exercise psychology. African Journal for Physical Health Education, Recreation and Dance, 20(2.1), 500-510.

Edwards, S. D. (2015). HeartMath: A positive psychology paradigm for promoting psychophysiological and global coherence. Journal of Psychology in Africa, 25(4), 367-374.

Goessl, V. C., Curtiss, J. E., \& Hofmann, S. G. (2017). The effect of heart rate variability biofeedback training on stress and anxiety: A meta-analysis. Psychological Medicine, 47(15), 2578-2586. https://doi.org/10.1017/S0033291717001003

Institute of HeartMath (2014). Building personal resilience. A handbook for HeartMath certified coachers and mentors. Boulder Creek, CA: Institute of HeartMath.

Kabat-Zinn, J. (2003). Mindfulness - based interventions in context: Past, present, and future. Clinical psychology: Science and Practice, 10(2), 144-156. https://doi.org/10.1093/clipsy.bpg016

Klepp, O. M., Mastekaasa, A., Sorensen, T., Sandanger, I., \& Kleiner, R. (2007). Structure analysis of Antonovsky's sense of coherence from an epidemiological mental health survey with a brief nine-item sense of coherence scale. International Journal of Methods in Psychiatric Research, 61(1), 11-22. https://doi.org/10.1002/mpr.197 
Laborde, S., Mosley, E., \& Thayer, J. F. (2017). Heart rate variability and cardiac vagal tone in psychophysiological research-recommendations for experiment planning, data analysis, and data reporting. Frontiers in Psychology, 8. https://doi.org/10.3389/fpsyg.2017.00213

Lemaire, J. B., Wallace, J. E., Lewin, A. M., de Grood, J., \& Schaefer, J. P. (2011). The effect of a biofeedback-based stress management tool on physician stress: A randomized controlled clinical trial. Open Med, 5(4), 154-163.

McCraty, R., Atkinson, M., Tomasino, D., \& Bradley, R. J. (2009). The coherent heart. Heart-brain interaction, psychophysiological coherence and the emergence of a system wide order. Integral Review, 2, 10-115.

McCraty, R., \& Shaffer, F. (2015). Heart rate variability: New perspectives on physiological mechanisms, assessment of self-regulatory capacity, and health risk. Global Advances in Health and Medicine, 4(1), 46-61. https://doi.org/10.7453/gahmj.2014.073

McCraty, R., \& Zayas, M. A. (2014). Cardiac coherence, self-regulation, autonomic stability and psychosocial well-being. Frontiers in Psychology, 5. https://doi.org/10.3389/fpsyg.2014.01090

McNair, D. M., Lorr, M., \& Doppleman, L. F. (1971). Manual for the Profile of Mood States. San Diego, CA: Educational and Industrial Testing Service.

Quintana, D. S., \& Heathers, J. A. (2014). Considerations in the assessment of heart rate variability in biobehavioral research. Frontiers in Psychology, 5. https://doi.org/10.3389/fpsyg.2014.00805

Ratanasiripong, P., Park, J. F., Ratanasiripong, N., \& Kathalae, D. (2015). Stress and anxiety management in nursing students: Biofeedback and mindfulness meditation. Journal of Nursing Education, 54(9), 520-524. https://doi.org/10.3928/01484834-20150814-07

Reiner, G., Atkinson, M., \& McCraty, R. (2008). The physiological and psychological effects of compassion and anger. Journal of Advancement in Medicine, 8(2), 87-105.

Seligman, M. E. P., \& Csikszentmihalyi, M. (2000). Positive psychology: An introduction. The American Psychologist, 55(1), 5-14. https://doi.org/10.1037/0003-066X.55.1.5

Tamres, L. K., Janicki, D., \& Helgeson, V. S. (2002). Sex differences in coping behavior: A meta-analytic review and an examination of relative coping. Personality and social psychology review, 6(1), 2-30. https://doi.org/10.1207/S15327957PSPR0601_1

van der Zwan, J. E., de Vente, W., Huizink, A. C., Bögels, S. M., \& de Bruin, E. I. (2015). Physical activity, mindfulness meditation, or heart rate variability biofeedback for stress reduction: a randomized controlled $\begin{array}{llll}\text { trial. Applied Psychophysiology and Biofeedback, 40(4), 257-268. } & \text {. }\end{array}$ https://doi.org/10.1007/s10484-015-9293-x

Walach, H., Buchheld, N., Buttenmüller, V., Kleinknecht, N., \& Schmidt, S. (2006). Measuring mindfulness - the Freiburg mindfulness inventory (FMI). Personality and Individual Differences, 40(8), 1543-1555. https://doi.org/10.1016/j.paid.2005.11.025

Whited, A., Larkin, K. T., \& Whited, M. (2014). Effectiveness of emWave biofeedback in improving heart rate variability reactivity to and recovery from stress. Applied Psychophysiology and Biofeedback, 39(2), 75-88. https://doi.org/10.1007/s10484-014-9243-z

\section{Copyrights}

Copyright for this article is retained by the author(s), with first publication rights granted to the journal.

This is an open-access article distributed under the terms and conditions of the Creative Commons Attribution license (http://creativecommons.org/licenses/by/4.0/). 